\title{
OPEN Population difference gratings created on vibrational transitions by nonoverlapping subcycle $\mathrm{THz}$ pulses
}

\author{
Rostislav Arkhipov ${ }^{1,2,3}$, Anton Pakhomov², Mikhail Arkhipov ${ }^{1,2}$, Ihar Babushkin ${ }^{4,5,6 \bowtie}$, \\ Ayhan Demircan ${ }^{4,5}$, Uwe Morgner ${ }^{4,5}$ \& Nikolay Rosanov ${ }^{2,3}$
}

We study theoretically a possibility of creation and ultrafast control (erasing, spatial frequency multiplication) of population density gratings in a multi-level resonant medium having a resonance transition frequency in the $\mathrm{THz}$ range. These gratings are produced by subcycle $\mathrm{THz}$ pulses coherently interacting with a nonlinear medium, without any need for pulses to overlap, thereby utilizing an indirect pulse interaction via an induced coherent polarization grating. High values of dipole moments of the transitions in the $\mathrm{THz}$ range facilitate low field strength of the needed $\mathrm{THz}$ excitation. Our results clearly show this possibility in multi-level resonant media. Our theoretical approach is based on an approximate analytical solution of time-dependent Schrödinger equation (TDSE) using perturbation theory. Remarkably, as we show here, quasi-unipolar subcycle pulses allow more efficient excitation of higher quantum levels, leading to gratings with a stronger modulation depth. Numerical simulations, performed for $\mathrm{THz}$ resonances of the $\mathrm{H}_{2} \mathrm{O}$ molecule using Bloch equations for density matrix elements, are in agreement with analytical results in the perturbative regime. In the strongfield non-perturbative regime, the spatial shape of the gratings becomes non-harmonic. A possibility of $\mathrm{THz}$ radiation control using such gratings is discussed. The predicted phenomena open novel avenues in THz spectroscopy of molecules with unipolar and quasi-unipolar THz light bursts and allow for better control of ultra-short THz pulses.

Generation of few-cycle $\mathrm{THz}$ pulses $(0.1-10 \mathrm{THz})$ have attracted considerable interest over the past decades ${ }^{1-4}$ due to the growing number of applications in science and technology. For example, $\mathrm{THz}$ pulses are used for spectroscopy, since the $\mathrm{THz}$ range includes rotational and vibrational resonances of large molecules as well as transitions in various dielectrics and semiconductor structures ${ }^{3-10}$. They have a huge potential in other applications such as medicine, wireless communication systems, charge particles acceleration etc ${ }^{3,4}$. Nowadays, $\mathrm{THz}$ pulses up to subcycle duration became available ${ }^{1-4}$. Such durations can be much shorter than the relaxation times $T_{1}$ and $T_{2}$ of the resonant medium. That is, so called coherent interaction regime takes place, and effects of resonant light-matter interactions such as Rabi oscillations may arise ${ }^{11}$. If a resonant medium interacts coherently with a train of few-cycle pulses and the pulses do not overlap in the medium, population density gratings can be created in a two-level medium as it was shown in optical range using long nanosecond ${ }^{12-14}$, femtosecond and attosecond pulses ${ }^{15-17}$ as well as in $\mathrm{THz}$ range using multi-cycle $\mathrm{THz}^{18}$ pulses.

Such gratings are based on interaction between the pulses without an actual overlap, taking place indirectly, via oscillations of macroscopic polarization of the medium. The first pulse prepares the medium in a coherent superposition of the ground and excited states. This superposition exists within the time scale of $T_{2}$, until destroyed by decoherence. If a second pulse enters the medium within this time, it interacts with this induced atomic polarization, and in this way an inversion grating can be formed. This is in contrast to the traditional approach in which such gratings are generated as a result of interference of two or more long quasimonochromatic overlapping beams ${ }^{19}$.

\footnotetext{
${ }^{1}$ St. Petersburg State University, Saint Petersburg, Russian Federation. ${ }^{2}$ ITMO University, Saint Petersburg, Russian Federation. ${ }^{3}$ Ioffe Institute, Saint Petersburg, Russian Federation. ${ }^{4}$ University of Hannover, Hannover, Germany. ${ }^{5}$ Cluster of Excellence PhoenixD (Photonics, Optics, and Engineering—Innovation Across Disciplines), Hannover, Germany. ${ }^{6}$ Max Born Institute, Berlin, Germany. ${ }^{\square}$ email: babushkin@iqo.uni-hannover.de
} 
The possibility of ultrafast creation and control of optical gratings by attosecond-long single-cycle and subcycle optical pulse trains was studied in ${ }^{15-17}$. However, generation of attosecond pulses with single-cycle and subcycle pulse duration requires a complicated setup. Besides, in the optical range, the field strengths needed to facilitate the effective interaction are extremely high $\left(10^{4}-10^{5} \mathrm{ESU} \approx 10^{6}-10^{7} \mathrm{~V} / \mathrm{cm}\right)$, which makes practical implementation even more complicated. In contrast, in the $\mathrm{THz}$ range, subcycle pulses can be generated much easier ${ }^{1-4}$.

Next, the pump power needed to realize coherent interactions in $\mathrm{THz}$ range is several orders of magnitude lower, provided that high THz vibrational transitions possess huge dipole moments $d_{12}{ }^{20,21}$. The latter allows using $\mathrm{THz}$ pulses experimentally available to date, and field strengths, significantly lower than in the optical range. It worth to note that practically available subcycle $\mathrm{THz}$ pulses often contain a burst of single polarity and a big tail of opposite polarity and small amplitude ${ }^{1-4,22-38}$. Action of such quasi-unipolar pulses is nearly the same as the true unipolar ones ${ }^{38}$.

Generation of unipolar and quasi-unipolar pulses and their applications in optics is a subject of active discussions ${ }^{22-30}$, see also recent review ${ }^{31}$ and references therein. These pulses, due to their unipolar character, effectively transfer their energy to charged particles, resulting in novel applications, including highly effective ionization and control of Rydberg atoms ${ }^{32-34}$, effective attosecond pulse generation ${ }^{35}$, effective excitation and ultra-fast control of electron wave packets dynamics ${ }^{23,36-38}$, charge acceleration ${ }^{39}$, holographic recording ${ }^{40}$ and $^{2}$ others.

As we mentioned above, in previous works of the authors ${ }^{15-17}$ grating dynamics was studied in the optical range using few-cycle pulses in a two-level medium. However, due to broad spectral content of few-cycle pulses taking into account multilevel character of the system is of high importance. Some remarks on the possibility of grating formation in multi-level systems in the optical range were shortly reported in a prior work ${ }^{16,41}$. However, a detailed analysis of grating dynamics under such circumstances was up to now still missing. In this paper, we study a possibility of population density grating formation on vibrational $\mathrm{THz}$ resonance transitions in three- and multi-level media, assuming high values of transition dipole moments (tens to hundreds Debyes), created by subcycle $\mathrm{THz}$ pulses. We consider a possibility to use short quasi-unipolar subcycle pulses for grating formation, because they allow more effective excitation and control of quantum state population ${ }^{23,36-38}$. In previous studies, only bipolar few-cycle pulses were considered in this context $\mathrm{t}^{15,16}$.

For the theoretical analysis of the multi-level system in question we use the standard perturbation approach for the time-dependent Schrödinger equation (TDSE) for "small" electric field amplitude. For numerical simulations, we use the Bloch equations for density matrix elements of a three-level medium. For analytical studies, we model the multi-level system as a quantum harmonic oscillator (HO) with equidistant levels assuming resonance frequency in $\mathrm{THz}$ range and high values of dipole moments of the resonant transitions. In spite of the fact, that our theoretical analysis is quite general, some particular examples will be mentioned.

To realize coherent light-matter interactions, the Rabi frequency $\Omega_{R}=d_{12} E_{0} / \hbar$ ( $d_{12}$ is the transition dipole moment, $E_{0}$ is the electric field amplitude) should be larger than the inverse polarization relaxation time $T_{2}$, $\Omega_{R}>1 / T_{2}$ and the pulse duration $\tau_{p}<T_{2}$. First, most of molecules have vibration resonances in THz range ${ }^{6-10}$ and low energy states can be modelled, to some level of approximation, using quantum $\mathrm{HO}^{42}$. In our analysis below we consider a water molecule $\mathrm{H}_{2} \mathrm{O}$ having resonances in the interval $0.5-1 \mathrm{THz}^{43,44}$. Other examples are Rydberg atoms with large quantum numbers $n>>1$ - they also have large dipole moments ${ }^{45}$, can be arranged to possess long life times ${ }^{46}$ and resonance transitions in $\mathrm{THz}$ range $\mathrm{e}^{32-34,45}$. Next, it is shown ${ }^{47}$ that the confinement potential of an electron in nanostructures and quantum dots (QD) for energy below the Fermi level is approximately parabolic, thus, the HO model can be used in this case. QD media can have extremely high values of transition dipole moments (tens-hundreds Debye) as well as $T_{2}$ at low temperatures as high as hundreds $\mathrm{ns}^{48,49}$; they also can be used for THz generation ${ }^{50}$. Furthermore, quantum cascade lasers can generate THz radiation ${ }^{51}$. Semiconductor nanostructures used in such devices have very high dipole moments (tens of Debye) and can operate as three-level systems ${ }^{52,53}$. In some media like crystals, containing impurities of rare-earth ions, the values of $T_{2}$ at low temperatures can approach extremely high values - from several seconds to several hours ${ }^{54}$.

There is another advantage of creating grating on vibrations transitions in THz range: In quantum $\mathrm{HO}$ in the first order perturbation approach, only the transition from the ground state to the 1st excited state is possible, in contrast to, for instance, $1 / r$ potential. As we see here, this significantly reduces the probability of excitation of higher order levels, even if we go into nonperturbative regime with HO.

Furthermore, creation of the gratings on vibrational transitions in different systems opens novel opportunities in $\mathrm{THz}$ ultra-fast spectroscopy. For instance, diffraction of a weak probe pulse on such gratings can be used for spectroscopic measurements of $T_{2}-$ a task which is otherwise proved to be quite difficult experimentally ${ }^{12-14}$. As we show below, such gratings can be also used for control of $\mathrm{THz}$ radiation, for instance ultrafast pulse reshaping using fast mirrors.

This paper is organized as follows. First, in "Classical picture of grating formation in a system of classical HOs", for a deeper understanding of the idea of grating formation, we consider aclassical picture of the gratings arising in a medium consisting of classical harmonic oscillators (HO). In "Multi-level vibration system: weak $\mathrm{THz}$ field strength" we consider a grating formation in a multi-level system using perturbation theory for TDSE, when the THz field strength is low. Here we use a $\delta$-pulse approximation, valid when the pulse duration is smaller than the inverse transition period of the medium. After this, pulses of finite duration are considered. In "Numerical simulations" we perform numerical simulations for three-level $\mathrm{HO}$ using parameters for $\mathrm{H}_{2} \mathrm{O}$ molecule. A possibility of THz radiation control by the gratings is considered in the "Control of long THz pulses". Finally, concluding remarks are drawn. 
THz pulse 4 THz pulse 1

THz pulse 2 THz pulse 3
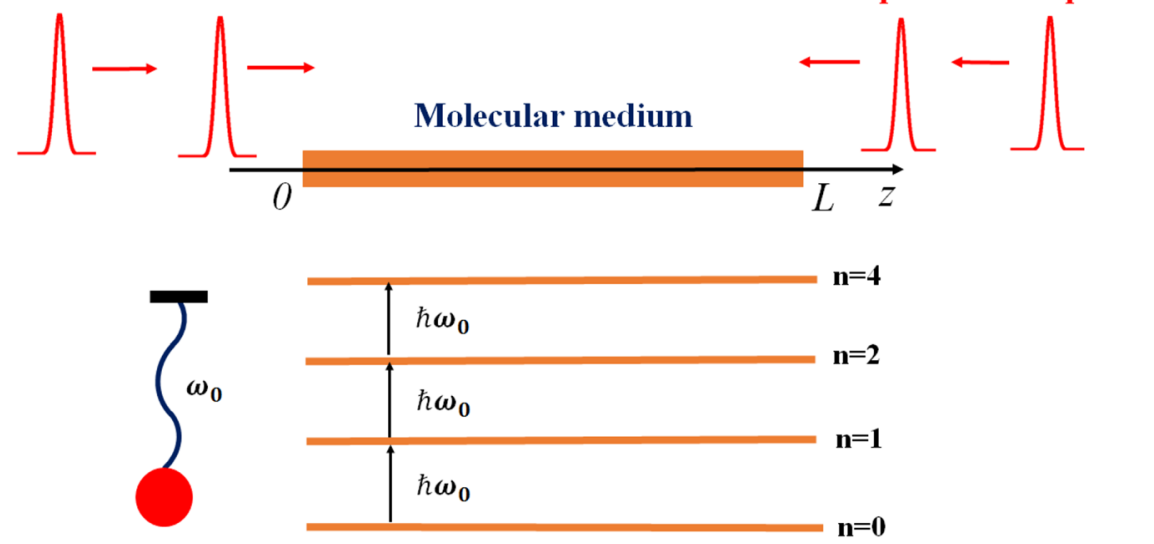

Figure 1. The simplest setup with four $\mathrm{THz}$ pulses counter-propagating in a medium to create a grating. Inset shows a 4-level $\mathrm{HO}$ molecular medium with the eigenfrequency $\omega_{0}$. This figure was created with Paint application for Windows and converted to eps using online convertor (https://image.online-convert.com/ru/ convert-to-eps).

\section{Classical picture of grating formation in a system of classical HOs}

First, we consider the classical picture of a grating formation. Let a dielectric resonant medium consist of linear classical harmonic oscillators, non-interacting with each other and having eigen-frequency $\omega_{0}$, distributed along $z$-axis, see Fig. 1. The displacement of the oscillator at point $z$ is governed by the following equation (neglecting damping of oscillations):

$$
\ddot{X}(z, t)+\omega_{0}^{2} X(z, t)=q / m E(z, t)
$$

Here $q$ is the electron charge and $m$ the oscillator mass. Let the medium be excited by a pair of extremely-short pulses with duration much smaller than the medium proper oscillations period $T_{0}=2 \pi / \omega_{0}$. In this approximation, and for simplicity assuming the electric field in the form of two delta pulses $E(t)=S_{0}[\delta(t)+\delta(t-\Delta)]$, counter-propagating as shown in Fig. 1, with the delay $\Delta(z)$ depending on the oscillator position $z$. We assume low enough density of the oscillators on the string, i.e., changing of the pulse shape during the propagation is negligible. Under the action of such kick-like excitation, the medium starts to harmonically oscillate at the eigenfrequency $\omega_{0}$. It can be easily shown that the response of the medium can be represented as ${ }^{55}$

$$
\begin{aligned}
X(z, t) & =X_{0} \sin \omega_{0} t, t<\Delta, \\
& =X_{0} \sin \omega_{0} t+X_{0} \sin \left(\omega_{0}(t-\Delta)\right)=X_{0} \cos \left(\omega_{0} \Delta / 2\right) \sin \left(\omega_{0} t+\omega_{0} \Delta / 2\right), t>\Delta .
\end{aligned}
$$

From the last expression it is seen that after arrival of the second pulse, the amplitude of the displacement periodically depends on the pulse-to-pulse delay $\cos \left(\omega_{0} \Delta / 2\right)$. This can be interpreted as periodic grating formation in the system of classical oscillators. In the next section, we consider the corresponding quantum picture.

\section{Multi-level vibration system: weak THz field strength}

Theoretical approach. In the previous studies we predicted population density gratings formation in a two-level medium ${ }^{15-17}$. However, subcycle pulses have broadband spectra and two-level approximation can be inapplicable. In this section we consider a multi-level vibration system, namely, a quantum harmonic oscillator (HO). The HO model is commonly used as a simplest model for theoretical description of molecular vibrations ${ }^{42}$.

Interaction of a subcycle pulse with a quantum system with arbitrary configuration of states is governed by the TDSE describing the evolution of the wave function $\psi^{42}$ :

$$
i \hbar \frac{\partial \psi}{\partial t}=\left[\hat{H}_{0}+V(t)\right] \psi
$$

$\hat{H}_{0}$ is the intrinsic Hamiltonian of the unperturbed system, and $V(t)=-q x E(t)$ is the interaction potential with the excitating pulse in the dipole approximation. The wave functions of the eigenstates are the eigenfunctions of the intrinsic Hamiltonian $\hat{H}_{0}$ and given by ${ }^{42}$ :

$$
\psi_{n}(x)=\left(\frac{m \omega_{0}}{\pi \hbar}\right)^{1 / 4} \frac{1}{\sqrt{2^{n} n !}} e^{-\frac{m \omega_{0} x^{2}}{2 \hbar}} H_{n}\left(x \sqrt{\frac{m \omega_{0}}{\hbar}}\right),
$$

where $H_{n}\left(x \sqrt{\frac{m \omega_{0}}{\hbar}}\right)$ - Hermite polynomials of order $n$. The general solution of the TDSE (3) can be written as a superposition of eigenstates $\psi_{n}$ with the amplitudes $a_{n}$ : 


$$
\psi(x, t)=\sum_{n} a_{n}(t) \psi_{n}(x)
$$

Let us assume that the system was initially in the ground state with $n=0$ and the pulse amplitude is small. The amplitudes of the eigenstates can be easily calculated in the 1 st order of the perturbation theor $\mathrm{y}^{42}$ :

$$
a_{n}^{I}=-\frac{i}{\hbar} \int V_{0 n} e^{i \omega_{0} t} d t
$$

The transition probability of a $\mathrm{HO}$ from the ground state of the discrete spectrum to the $n$-th state $w_{0 n}$ is given by $\left|a_{n}^{I}\right|^{242}$

$$
w_{0 n}=\frac{1}{\hbar^{2}}\left|\int V_{0 n} e^{i \omega_{0} t} d t\right|^{2} .
$$

$V_{0 n}$ is the matrix element of the perturbation operator, which is expressed through the matrix element of the dipole moment $d_{n, n+1}$ as:

$$
V_{n, n+1}=-d_{n, n+1} E(t) .
$$

For HO, the matrix $d_{n, n+1}$ is given by ${ }^{42}$ :

$$
d_{n, n+1}=q \sqrt{\frac{\hbar n(n+1)}{2 m \omega_{0}}} .
$$

Other matrix elements are zero, because in HO only transitions between neighbouring states are possible. That is, in the 1st order perturbation approach, only transition from the ground state to the 1st excited state has nonzero probability, i.e.,

$$
w_{01}=\frac{q^{2}}{2 m \omega_{0} \hbar}\left|\int E(t) e^{i \omega_{0} t} d t\right|^{2} .
$$

This allows us to consider $\mathrm{HO}$ as an effective two-level medium, as least in perturbative approximation. This gives rise to high-contrast grating formation only on the first transition $0-1$ of the vibrational spectrum.

Population density grating formation and their control using $\delta$-pulses. Before providing detailed analysis of the grating dynamics let us consider for simplicity interaction of a $\mathrm{HO}$ with a $\delta$-pulse. A validity of this approximation follows from the fact that experimentally obtained subcycle pulse shape contains a short burst of electric field (half-wave) of one polarity and a long damped tail of small amplitude of the opposite polarity ${ }^{1-4,22-30}$. In view of the small amplitude of the tail, the main contribution is given by the strong half-wave part $^{38}$. Assuming, that the pulse duration is smaller than all resonant transition periods in the atom/molecule, we can describe our driving field as a set of delta-function-like pulses:

$$
E(t)=\sum_{n} S_{E, n} \delta\left(t-\Delta_{n}\right)
$$

having the time delays $\Delta_{n}$ and electric pulse area $S_{E, n}=\int E_{n}(t) d t^{56}, E_{n}$ is the electric field of the $n$-th pulse.

In the case of a spatially extended medium, one can consider a string of atoms distributed along the $z$ axis. If the concentration of atoms in such a string is relatively small, one may neglect nonlinear pulse shape modification due to propagation effects. A setup for a grating formation in the case with four pulses is shown in Fig. 1 ( similar scheme was used earlier for a two-level medium ${ }^{15,16}$ ). The first pulse propagates in the medium from left to right, whereas the second one travels from right to left, after the 1st one already left the medium, so that the pulses do not overlap in the medium. Under these conditions, the delay between two pulses is constantly changing with time. The $3 \mathrm{rd}$ pulse propagates after the 2 nd one in the same direction. The 4 th pulse travels in the same direction as the 1 st one after 3rd pulse left the medium. To track this situation without directly solving the wave equation, we may consider every point separately. In such consideration, the difference from one point to another in $z$-direction is mapped to the delay between two pulses $\Delta \sim z / c$. Thus, we can consider a single-atom response and calculate the populations of the levels in dependence on the delay between two ultra-short pulses in order to show the existence of a grating as well as to estimate its properties.

First, consider the action of two pulses propagating in the opposite directions as shown in Fig. 1:

$$
E(t)=S_{0}[\delta(t)+\delta(t-\Delta)]
$$

where $\Delta$ is the delay between two pulses. Using Eq. (7) and performing integration it is easy to obtain an expression for the transition probability $w_{01}$ (the only nonzero one, according to Eq. (8)):

$$
w_{01}=2 \frac{d_{01}^{2} S_{0}^{2}}{\hbar^{2}}\left(1+\cos \omega_{01} \Delta\right)
$$

From Eq. (12), the periodic dependence of the transition probability on the delay between the pulses $\Delta$ is seen. Since in the case of an extended medium, the delay $\Delta \sim z / c$ determines the time of arrival of the second pulse to a point of the medium with coordinate $z$, Eq. (12) shows a possibility of a periodic grating formation in the 
medium. Equation (12) is valid for any multi-level quantum system, when the concentration of particles is small and the change in the pulse shape during the propagation process can be neglected. However, formula Eq. (12) was obtained in the perturbation theory in the weak-field approximation. The case of an arbitrary strong driving field will be considered below.

We now show a possibility of erasing and spatial frequency multiplication of the gratings. Suppose that the system interacts with three pulses, see Fig. 1:

$$
E(t)=S_{0}\left[\delta(t)+\delta(t-\Delta)+\delta\left(t-\Delta-\Delta_{23}\right)\right],
$$

where $\Delta_{23}$ is the delay between the 2nd and the 3rd pulse. Using Eq. (7) for the transition probability it is easy to obtain

$$
w_{01}=\frac{d_{01}^{2} S_{0}^{2}}{\hbar^{2}}\left|1+e^{i \omega_{01} \Delta}+e^{i \omega_{01} \Delta} e^{i \omega_{01} \Delta_{23}}\right|^{2} .
$$

From Eq. (14) it is seen that when $e^{i \omega_{01} \Delta_{23}}=-1$, the 3 rd pulse erases the grating since the probability of transition does not depend on the delay and thus on the spatial position.

Next, let the system interact with the 4 th pulse (see Fig. 1):

$$
E(t)=S_{0}\left[\delta(t)+\delta(t-\Delta)+\delta\left(t-\delta-\Delta_{23}\right)+\delta\left(t-\Delta_{23}-\Delta_{34}\right)\right],
$$

where $\Delta_{34}$ - delay between the $3 \mathrm{rd}$ and the 4 th pulse. For the transition probability we obtain:

$$
w_{01}=\frac{d_{01}^{2} S_{0}^{2}}{\hbar^{2}}\left|1+e^{i \omega_{01} \Delta}+e^{i \omega_{01} \Delta} e^{i \omega_{01} \Delta_{23}}+e^{i \omega_{01} \Delta} e^{i \omega_{01} \Delta_{23}} e^{i \omega_{01} \Delta_{34}}\right|^{2} .
$$

We now show the possibility of the multiplication of the spatial frequency of the gratings analogously as it is done in a two-level system ${ }^{15}$. We choose the following values of the delays between pulses: $\Delta_{23}=\pi / \omega_{01}+2 \pi k / \omega_{01}$, that is $e^{i \omega_{01} \Delta_{23}}=-1$ ) and $\Delta_{34}=\Delta+2 \pi k / \omega_{01}$, where $k=0,2,4,6$ Hence, from Eq. (16) it is easy to obtain:

$$
w_{01}=\frac{d_{01}^{2} S_{0}^{2}}{\hbar^{2}}\left|1-e^{2 i \omega_{01} \Delta}\right|^{2}=2 \frac{d_{01}^{2} S_{0}^{2}}{\hbar^{2}}\left(1-\cos 2 \omega_{01} \Delta\right) \text {. }
$$

From Eq. (17) it is seen that the spatial frequency of the gratings is multiplied by a factor of two. Thus, we showed that by selecting the delay between the pulses it is possible to control period of the multi-level-based grating.

Population density grating formation using subcycle $\mathrm{THz}$ pulses of finite duration. Using Eq. (7), we show a possibility of existence of population density gratings in HO-type systems for the pulses of finite duration. As we mentioned above, experimentally obtained half-cycle THz pulses contain a strong halfwave of high amplitude and a long weak tail of the opposite polarity ${ }^{1-4}$. It was shown $\mathrm{in}^{38}$, that the impact of this tail can be neglected, provided that its amplitude is small, and duration is larger than the width of the main half-wave. Hence, we first neglect for simplicity this tail and consider the impact on the system of two Gaussian pulses given by:

$$
E(t)=E_{0} \exp \left(-t^{2} / \tau_{p}^{2}\right)+E_{0} \exp \left(-(t-\Delta)^{2} / \tau_{p}^{2}\right)
$$

where $\Delta$ is the time delay between two pulses. In the subsequent analysis we include the tail into consideration. Substituting Eq. (18) into Eq. (7) and taking account that $\int e^{i b x-a x^{2}} d x=\sqrt{\frac{\pi}{a}} e^{\frac{-b^{2}}{4 a}}$, we obtain

$$
w_{01}=\frac{2 \pi q^{2} E_{0}^{2} \tau_{p}^{2}}{m \hbar \omega_{0}} \exp \frac{-\omega_{0}^{2} \tau_{p}^{2}}{2}\left(1+\cos \omega_{0} \Delta\right),
$$

where we have explicitly used Eq. (8) for $d_{01}$ as well as denoted the transition frequency $0 \rightarrow 1$ as $\omega_{0}=\omega_{01}$. Expression Eq. (19) is proportional to the square of the field strength $E_{0}$ and at the first glance it seems that probability $w_{01}$ can be very high at large field amplitudes. However, Eq. (19) was obtained using perturbation approach and is valid when the electric field amplitude is small, i.e. $q^{2} E_{0}^{2} \pi \tau_{p}^{2} / m \ll \hbar \omega_{0}$. From Eq. (19) one can also see that the periodic dependence of the transition probability (population of the first excited state) on the delay between pulses $\Delta$ is similar to the case of $\delta$-function-like pulses considered earlier. The modulation depth of the grating is proportional to the square of the electric field amplitude of the pulse. Formula Eq. (19) can be interpreted as a harmonic inversion grating created by a pair of counter-propagating subcycle pulses in a spatially-extended medium. Thus, in the case of the pulses with finite duration the existence of the inversion gratings is also possible.

We apply the theory developed above to a $\mathrm{H}_{2} \mathrm{O}$ molecule. It has isolated resonances in the $\mathrm{THz}$ range, in particular, around $0.97-0.99 \mathrm{THz}$ according to experimental results of ${ }^{43}$. Below we take $\omega_{0} / 2 \pi \approx 0.97 \mathrm{THz}$. Using the value for the "characteristic radius" of water $R=4$ Angstrom $^{44}$, we estimate transition dipole moment as $d_{01}=q R=19.2$ Debye ( $q$ is the electron charge). The two-dimensional diagram in Fig. 2 illustrates the dependence of the 1st excited state population $w_{01}$ Eq. (19) for water molecules vs the pulse duration $\tau_{p}$ and the pulse amplitude $E_{0}$ for $\Delta=3 \mathrm{fs}$. The maximal probability value $w_{01}$ at the fixed delay $\Delta$ is determined by the term $\tau_{p}^{2} \exp \frac{-\omega_{0}^{2} \tau_{p}^{2}}{2}$. For small pulse durations (shorter than the transition period, $\omega_{0} \tau_{p} \ll 1$ ) this term increases with increase of $\tau_{p}$, reaching its maximum value at certain point. With the particular parameters mentioned above, $w_{01}$ reaches its maximum at the pulse duration $\tau_{p}$ of around $200 \mathrm{fs}$. When the pulse duration becomes larger than 


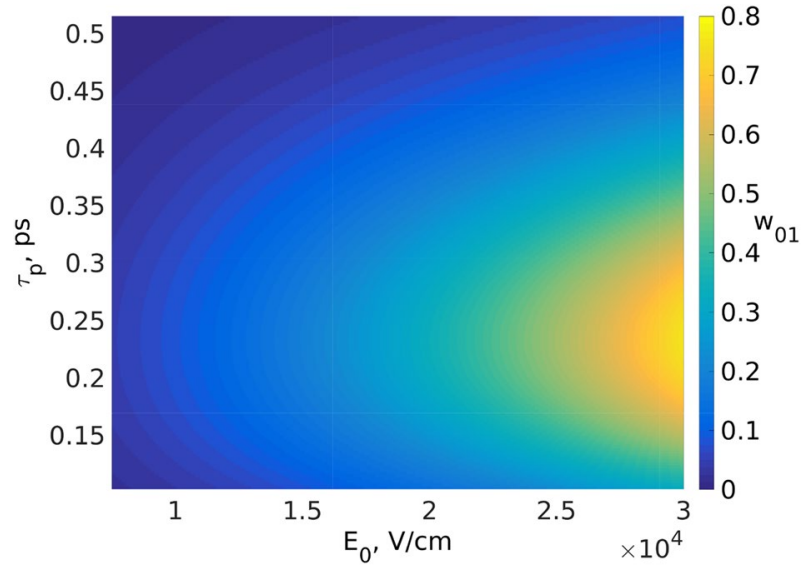

Figure 2. Dependence of the population of the 1st excited state $w_{01}$ on the pulse duration $\tau_{p}$ and the amplitude $E_{0}$ calculated for $\mathrm{H}_{2} \mathrm{O}$ molecule. Other parameters: $\Delta=3 \mathrm{ps}, \omega_{0} / 2 \pi=0.97 \mathrm{THz}, d_{01}=19.2$ Debye. This figure was created with Matlab R2016b (www.mathworks.com).

the transition period, $\omega_{0} \tau_{p} \gg 1$, the population $w_{01}$ tends to zero. It means, that an unipolar pulse with a duration smaller than the inverse transition frequency $\left(\omega_{0} \tau_{p} \ll 1\right)$ acts more effectively than a long unipolar one with a larger duration and the same amplitude. This statement is valid in general case of quasi unipolar ones, see below.

It can be also seen, that the modulation depth of the gratings significantly depends on the duration of the incident pulses and their amplitudes. It is also seen from Fig. 2 that for creation of gratings, electric fields of small amplitude of $\sim \mathrm{kV} / \mathrm{cm}$ are sufficient, what can be easily reached in practice ${ }^{1-4}$.

Since the HO model is used to describe molecular vibrations (more precisely, low excited vibration states), and the oscillation frequencies of the molecules could lie in the THz region, the analytical result Eq. (19) clearly shows the possibility of a grating creating in molecular systems using a pair of THz subcycle pulses with experimentally available strengths, several orders of magnitude lower than in the optical range.

Now, we proceed further to take into account the tails of the Gaussian pulses. We assume, instead of Eq. (18), the following more general expression for the exciting field:

$$
E(t)=E_{0} \exp \left(-t^{2} / \tau_{p}^{2}\right) \cos \left(\Omega t+\varphi_{1}\right)+E_{0} \exp \left(-(t-\Delta)^{2} / \tau_{p}^{2}\right) \cos \left(\Omega(t-\Delta)+\varphi_{2}\right),
$$

with the carrier-wave frequency $\Omega$ and the carrier-envelope phase (CEP) $\varphi_{i}$. For clarity, we assume below CEP for all pulses in the sequence to be fixed $\varphi_{i}=\varphi=$ const, i.e., that the pulses are coming from a CEP-stabilized laser pulse source. The electric pulse area for such pulses is given as:

$$
S_{E}=\sqrt{\pi} E_{0} \tau_{p} \exp \left(-\Omega^{2} \tau_{p}^{2} / 4\right) \cos \varphi,
$$

so that these pulses can be safely treated as unipolar ones if $\Omega \tau_{p} \sim 1$ or even $\Omega \tau_{p} \ll 1$ and $\varphi \neq \pm \pi / 2$.

Substituting now Eq. (20) into Eq. (7) and performing integration we find:

$$
w_{01}=\frac{\pi q^{2} E_{0}^{2} \tau_{p}^{2}}{m \hbar \omega_{0}} \exp \frac{-\left(\omega_{0}^{2}+\Omega^{2}\right) \tau_{p}^{2}}{2}\left(\cosh \left(\omega_{0} \Omega \tau_{p}^{2}\right)+\cos 2 \varphi\right)\left(1+\cos \omega_{0} \Delta\right) .
$$

Equation (22) again yields a periodic dependence of the transition probability $w_{01}$ (population of the first excited state) on the delay $\Delta$ similarly to Eq. (19) above. Moreover, Eq. (19) contains some interesting peculiarities. When $\operatorname{CEP} \varphi= \pm \pi / 2$, i.e. the exciting pulses are strictly bipolar according to Eq. (21) and $S_{E}=0$, the population gratings still arise, even though they have smaller modulation depth. The dependence of the modulation amplitude on the duration and CEP of the pump pulses is determined from Eq. (22) by the following factor:

$$
\kappa=\omega_{0}^{2} \tau_{p}^{2} \exp \frac{-\left(\omega_{0}^{2}+\Omega^{2}\right) \tau_{p}^{2}}{2}\left(\cosh \left(\omega_{0} \Omega \tau_{p}^{2}\right)+\cos 2 \varphi\right),
$$

where we have introduce the factor $\omega_{0}^{2}$ to make the parameter $\kappa$ dimensionless. Figure 3 illustrates the dependence of this parameter on $\operatorname{CEP} \varphi$ and the pulse duration $\tau_{p}$. One can see that $\kappa$ achieves its maximal value at $\tau_{p} \approx 0.4$ ps and $\cos \varphi=1$. Also, it is worth noting that in the limit $\Omega \rightarrow 0$ Eq. (22) coincides with Eq. (12).

It is also seen from Fig. 3 that the grating modulation depth is larger for subcycle pulses $\left(\Omega \tau_{p} \ll 1\right)$ than for multi-cycle ones, and it is smaller for bipolar pulses (when $\varphi=\pi / 2$ and the pulse area $S_{E}=0$ ). This fact is in agreement with our previous results, which showed that quasi-unipolar subcycle pulses allow more efficient nonresonant excitation of quantum systems with respect to single-cycle and multi-cycle ones ${ }^{37,38}$.

Let us check now the results of the previous section for the sequence of three and four pump pulses. For three pulses we have the exciting electric field in the form: 


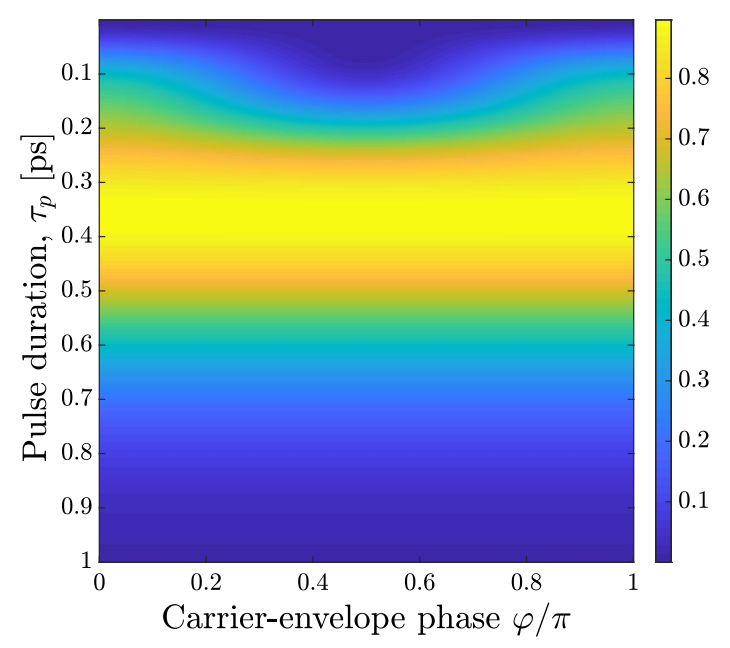

Figure 3. Dependence of the parameter $\kappa$ from Eq. (23) on the carrier-envelope phase (CEP) $\varphi$ and the pulse duration $\tau_{p}$; other parameters: $\omega_{0} / 2 \pi=0.97 \mathrm{THz}, \Omega=2 \cdot 10^{12} \mathrm{~s}^{-1}$. This figure was created with Matlab R2018a (www.mathworks.com).

$$
\begin{aligned}
E(t)= & E_{0} \exp \left(-t^{2} / \tau_{p}^{2}\right) \cos (\Omega t+\varphi)+E_{0} \exp \left(-(t-\Delta)^{2} / \tau_{p}^{2}\right) \cos (\Omega(t-\Delta)+\varphi) \\
& +E_{0} \exp \left(-\left(t-\Delta-\Delta_{23}\right)^{2} / \tau_{p}^{2}\right) \cos \left(\Omega\left(t-\Delta-\Delta_{23}\right)+\varphi\right),
\end{aligned}
$$

and Eq. (7) gives:

$$
\begin{gathered}
w_{01}=\frac{\pi q^{2} E_{0}^{2} \tau_{p}^{2}}{2 m \hbar \omega_{0}} \exp \frac{-\left(\omega_{0}^{2}+\Omega^{2}\right) \tau_{p}^{2}}{2}\left(\cosh \left(\omega_{0} \Omega \tau_{p}^{2}\right)+\cos 2 \varphi\right) \\
\times\left(3+2 \cos \omega_{0} \Delta+2 \cos \omega_{0}\left(\Delta+\Delta_{23}\right)+2 \cos \omega_{0} \Delta_{23}\right) .
\end{gathered}
$$

If we choose $\Delta_{23}=\pi / \omega_{0}+2 \pi n \omega_{0}$, Eq. (24) reduces to:

$$
w_{01}=\frac{\pi q^{2} E_{0}^{2} \tau_{p}^{2}}{2 m \hbar \omega_{0}} \exp \frac{-\left(\omega_{0}^{2}+\Omega^{2}\right) \tau_{p}^{2}}{2}\left(\cosh \left(\omega_{0} \Omega \tau_{p}^{2}\right)+\cos 2 \varphi\right)
$$

i.e., the third pulse erases the population grating and we get a constant value of $w_{01}$ along the medium. Finally, when we add the fourth pulse:

$$
\begin{aligned}
E(t)= & E_{0} \exp \left(-t^{2} / \tau_{p}^{2}\right) \cos (\Omega t+\varphi)+E_{0} \exp \left(-(t-\Delta)^{2} / \tau_{p}^{2}\right) \cos (\Omega(t-\Delta)+\varphi) \\
& +E_{0} \exp \left(-\left(t-\Delta-\Delta_{23}\right)^{2} / \tau_{p}^{2}\right) \cos \left(\Omega\left(t-\Delta-\Delta_{23}\right)+\varphi\right) \\
& +E_{0} \exp \left(-\left(t-\Delta-\Delta_{23}-\Delta_{34}\right)^{2} / \tau_{p}^{2}\right) \cos \left(\Omega\left(t-\Delta-\Delta_{23}-\Delta_{34}\right)+\varphi\right),
\end{aligned}
$$

and we obtain with Eq. (7):

$$
\begin{aligned}
w_{01}= & \frac{\pi q^{2} E_{0}^{2} \tau_{p}^{2}}{2 m \hbar \omega_{0}} \exp \frac{-\left(\omega_{0}^{2}+\Omega^{2}\right) \tau_{p}^{2}}{2}\left(\cosh \left(\omega_{0} \Omega \tau_{p}^{2}\right)+\cos 2 \varphi\right)\left(4+2 \cos \omega_{0} \Delta+2 \cos \omega_{0}\left(\Delta+\Delta_{23}\right)\right. \\
& \left.+2 \cos \omega_{0} \Delta_{23}+2 \cos \omega_{0}\left(\Delta+\Delta_{23}+\Delta_{34}\right)+2 \cos \omega_{0}\left(\Delta_{23}+\Delta_{34}\right)+2 \cos \omega_{0} \Delta_{34}\right) .
\end{aligned}
$$

As we choose the delays between pulses: $\Delta_{23}=\pi / \omega_{0}+2 \pi n / \omega_{0}$ and $\Delta_{34}=\Delta+2 \pi n / \omega_{0}$ with integer $n$, we get the following population grating:

$$
w_{01}=\frac{\pi q^{2} E_{0}^{2} \tau_{p}^{2}}{m \hbar \omega_{0}} \exp \frac{-\left(\omega_{0}^{2}+\Omega^{2}\right) \tau_{p}^{2}}{2}\left(\cosh \left(\omega_{0} \Omega \tau_{p}^{2}\right)+\cos 2 \varphi\right)\left(1-\cos 2 \omega_{0} \Delta\right),
$$

i.e., the spatial frequency of the grating is multiplied by factor of 2 . 


\section{Numerical simulations}

To check our analytical theory and observe, what happens in the nonperturbative regime, we solve numerically a system of Bloch equations for density-matrix element, which models interaction of a three-level medium with THz pulses:

$$
\begin{gathered}
\frac{\partial}{\partial t} \rho_{21}=-\rho_{21} / T_{21}-i \omega_{21} \rho_{21}-i \frac{d_{21} E}{\hbar}\left(\rho_{22}-\rho_{11}\right)-i \frac{d_{13} E}{\hbar} \rho_{32}+i \frac{d_{23} E}{\hbar} \rho_{31}, \\
\frac{\partial}{\partial t} \rho_{32}=-\rho_{23} / T_{32}-i \omega_{32} \rho_{32}-i \frac{d_{32} E}{\hbar}\left(\rho_{33}-\rho_{22}\right)-i \frac{d_{21} E}{\hbar} \rho_{31}+i \frac{d_{13} E}{\hbar} \rho_{21}, \\
\frac{\partial}{\partial t} \rho_{31}=-\rho_{31} / T_{31}-i \omega_{31} \rho_{31}-i \frac{d_{31} E}{\hbar}\left(\rho_{33}-\rho_{11}\right)-i \frac{d_{21} E}{\hbar} \rho_{32}+i \frac{d_{23} E}{\hbar} \rho_{21}, \\
\frac{\partial}{\partial t} \rho_{11}=\rho_{22} / T_{22}+\rho_{33} / T_{33}+i \frac{d_{21} E}{\hbar}\left(\rho_{21}-\rho_{21}^{*}\right)-i \frac{d_{13} E}{\hbar}\left(\rho_{13}-\rho_{13}^{*}\right), \\
\frac{\partial}{\partial t} \rho_{22}=-\rho_{22} / T_{22}-i \frac{d_{21} E}{\hbar}\left(\rho_{21}-\rho_{21}^{*}\right)-i \frac{d_{23} E}{\hbar}\left(\rho_{23}-\rho_{23}^{*}\right), \\
\frac{\partial}{\partial t} \rho_{33}=-\rho_{33} / T_{33}+i \frac{d_{13} E}{\hbar}\left(\rho_{13}-\rho_{13}^{*}\right)+i \frac{d_{23} E}{\hbar}\left(\rho_{23}-\rho_{23}^{*}\right) .
\end{gathered}
$$

Equations (27)-(29) describe the evolution of off-diagonal elements of the density matrix $\rho_{21}, \rho_{32}, \rho_{31}$, which are associated with the polarization of the medium. Equations (30)-(32) describe the evolution of diagonal elements of the density matrix $\rho_{11}, \rho_{22}, \rho_{33}$, which have the meaning of populations of the first, second and third levels, correspondingly. Parameters $d_{21}, d_{23}, d_{13}$ are the dipole moments of the corresponding transitions, $\omega_{21}, \omega_{32}, \omega_{31}$ are the transition frequencies. In our case, we consider a three-level HO medium, so $\omega_{21}=\omega_{32}=\omega_{0}$ and $\omega_{31}$ $=2 \omega_{0}, d_{13}=0$. Equations (27)-(29) also contain relaxation times of the non-diagonal elements of the density matrix $T_{21}, T_{32}, T_{31}$, and population lifetimes of the 2 nd and $3 \mathrm{rd}$ levels respectively $T_{22}$ and $T_{33}$. We performed series of numerical simulations for both low and high $\mathrm{THz}$ field amplitudes.

Low-power THz fields. Equation (19) was obtained using perturbation approach valid for low field strength. In the 1 st order perturbation approach, only probability transition $w_{01}$ is non-vanishing. This is because transition dipole moments in $\mathrm{HO} d_{0, n}=0$ for $n$ larger than 1. Numerical simulations (see below) showed that populations of higher levels are 10-100 times lower than the populations of the 2 nd level $w_{01}$. This fact enables the possibility of creating the high contrast grating on the main transition $0 \rightarrow 1$ neglecting other transitions using weak $\mathrm{THz}$ field values.

To confirm this statement, we performed numerical simulations using system of density matrix equations (27)-(32) with a pair of Gaussian pulses (18). Figure 4 shows the behaviour of the populations $\rho_{11}$ (a), $\rho_{22}$ (b), and $\rho_{33}$ (c) versus the delay $\Delta$ for the water molecule and $E_{0}=15 \mathrm{kV} / \mathrm{cm}$ assuming infinite relaxation times. The influence of finite relaxation times will be studied in the next section. It can be seen from Fig. $4 \mathrm{~b}$ that the grating obtained numerically has the same shape and roughly the same amplitude as predicted by Eq. (19). Besides, the population of the 3 rd level $\rho_{33}$ is 10 times smaller than $\rho_{32}$.

High-power THz fields. To study the grating dynamics in the high-power field we performed a series of the numerical simulations of the Bloch equations described above at different pump field strengths and durations. Figure 5 shows the behaviour of the populations difference $\rho_{11}-\rho_{22}$ (a), $\rho_{11}-\rho_{33}(\mathrm{~b}), \rho_{22}-\rho_{33}$ (c) versus the delay $\Delta$ between two Gaussian THz pulses and time $t$ for the $H_{2} 0$ molecule $\left(\omega_{0} / 2 \pi=0.97 \mathrm{THz}, d_{12}=19.2\right.$ Debye) for the following parameters: $E_{0}=150 \mathrm{kV} / \mathrm{cm}, \tau_{p}=150$ fs. Typical lifetimes of the vibrational levels $T_{1}$ and coherence times $T_{2}$ of vibration states are in the ps range ${ }^{57,58}$. We take relaxation population lifetimes $T_{22}=T_{33}=250 \mathrm{ps}$ and coherence lifetimes $T_{21}=T_{32}=T_{31}=5 \mathrm{ps}^{57,58}$.

One can see noticeable differences to the case of low field strength described above. The grating shape is not harmonic anymore, and has complex peak structure in contrast to the weak-field case. Also in contrast to the case of the two-level system, where both analytical and numerical solutions predict harmonic shape only ${ }^{15,16}$. Next, due to finite values of the relaxation times, the grating modulation depth decreases dramatically with time.

To summarize the results of these sections, we see that using $\mathrm{THz}$ pulses and vibrational transitions introduces many differences and advantages with respect to the previously studied case of optical field excitation. It allows reducing field strength using experimentally available subcycle $\mathrm{THz}$ fields. One can use only the transition $0 \rightarrow 1$ for grating formation in the weak-field case. The shape of the grating can be controlled by increasing the field strength, in contrast to the case of a two-level medium, where grating shape was always harmonic.

\section{Control of long THz pulses}

In this section, we describe an application of the gratings described above, to control propagation of $\mathrm{THz}$ pulses. As it follows from the Bloch equations, the modification of polarization in the linear regime corresponding to a density grating of full contrast $\left(\left|\rho_{22}-\rho_{11}\right|=2\right)$ is 

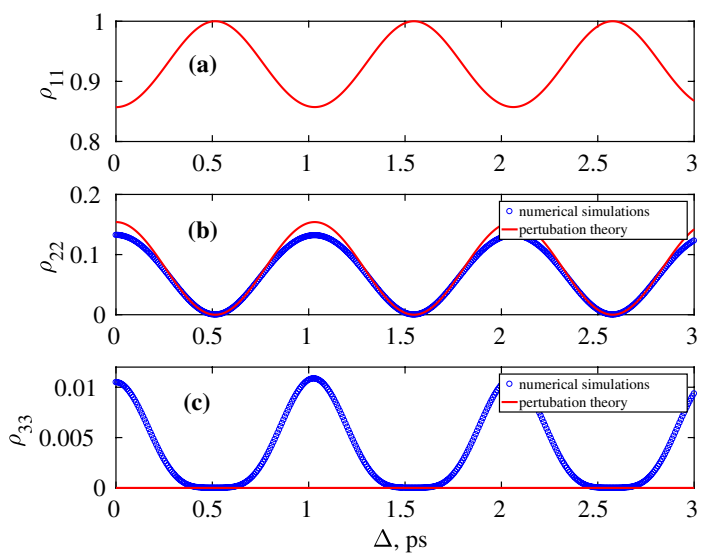

Figure 4. Dependence of the populations $\rho_{11}(\mathbf{a}), \rho_{22}(\mathbf{b})$, and $\rho_{33}(\mathbf{c})$ on the delay $\Delta$ between two Gaussian $\mathrm{THz}$ pulses for the $\mathrm{H}_{2} \mathrm{O}$ molecule. Blue circles show the result of the numerical simulations, red solid line show the analytical results obtained from Eq. (19). Parameters: $E_{0}=15000 \mathrm{~V} / \mathrm{cm}, \tau_{p}=150 \mathrm{fs}, d_{12}=19.2$ Debye, $\omega_{0} / 2 \pi=0.97 \mathrm{THz}$, all the relaxation times $T_{i k}=\infty$. This figure was created with Matlab R2016b (www.mathw orks.com).
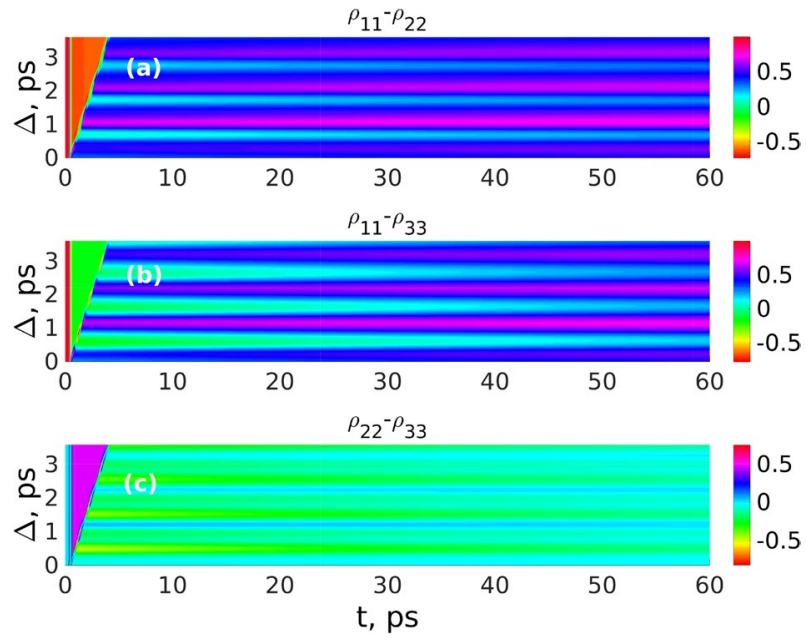

Figure 5. Dependence of the populations difference $\rho_{11}-\rho_{22}(\mathbf{a}), \rho_{11}-\rho_{33}(\mathbf{b}), \rho_{22}-\rho_{33}(\mathbf{c})$ on the delay $\Delta$ between two Gaussian THz pulses and time $t$ for parameters of the $H_{2} 0$ molecule $\left(\omega_{0} / 2 \pi=0.97 \mathrm{THz}\right.$, $d_{12}=19.2$ Debye). Other parameters: $E_{0}=150 \mathrm{kV} / \mathrm{cm}, \tau_{p}=150 \mathrm{fs}, T_{22}=T_{33}=250 \mathrm{ps}, T_{21}=T_{32}=T_{31}=5$ ps. This figure was created with Matlab R2016b (www.mathworks.com).

$$
|\delta P|=2 T_{2} d_{12}^{2} N E / \hbar,
$$

where $N$ is the atomic density. Taking into account that $\delta P=\varepsilon_{0} \chi E$, we have $|\chi|=2 d_{12}^{2} N T_{2} /\left(\hbar \varepsilon_{0}\right)$, and therefore the refractive index modification

$$
\delta n \approx \delta \chi / 2=\frac{d_{12}^{2} T_{2} N}{\varepsilon_{0} \hbar} .
$$

A refractive index grating is a powerful tool to make highly frequency-selective reflection of $\mathrm{THz}$ radiation, fully controllable on the single-cycle (picosecond) time scale. As an example, in Fig. 6 we show the dependence of reflection on a wavelength resulting from a grating containing 1000 oscillation periods, each having the length $75 \mu \mathrm{m}$ in a gas with atmospheric pressure consisting of active molecules, which thus creates a density grating with the contrast $\delta n \approx 10^{-3}$. The reflection is calculated using the standard multilevel approach ${ }^{59}:$ The ABCD matrix of a single layer of size $h$ for the normal incidence is

$$
M(h)=\left(\begin{array}{cc}
\cos \left(k_{0} n h\right) & -\frac{i}{p} \sin \left(k_{0} n h\right) \\
-i p \sin \left(k_{0} n h\right) & \cos \left(k_{0} n h\right)
\end{array}\right),
$$




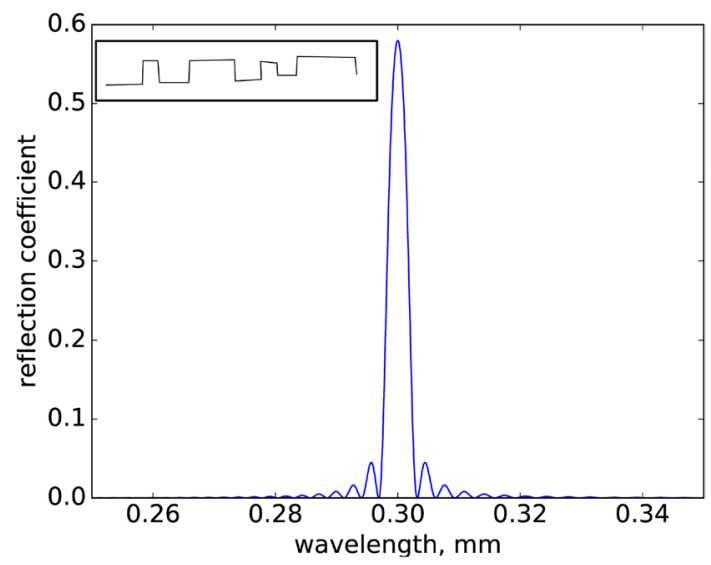

Figure 6. Reflection of a long weak narrow-line probe pulse in dependence on its wavelength from a grating containing 1000 periods ( $75 \mu \mathrm{m}$ each) in a medium with 1 bar pressure of the active molecules. The inset shows schematically a CW wave modulated by switching on and off the grating dynamically as a function of time. This figure was created in Phython 2.7, preinstalled with Ubuntu linux 18.04.05 (https://ubuntu.com/).

where $k_{0}$ is the wavevector in vacuum, $n$ is the refractive index, $p=\sqrt{\epsilon}$. For our calculation we have taken $\lambda / 4$ layers with interleaving layers of the refractive index $n_{1}=1-\delta n / 2$ and $n_{1}=1+\delta n / 2$. Multiplying the matrices for all layer gives the resulting reflection. Although an analytic expression exists for the whole such product ${ }^{59}$, we find more useful direct numerical multiplication.

The refractive index grating can be used in various ways. The key advantage is a possibility of fast switching on the single-cycle level. An example shown in the inset to Fig. 6 is a fast amplitude modulation of a THz CW wave allowing to encode information into it.

\section{Conclusions}

To summarize, we studied theoretically the possibility of population density grating formation created by subcycle $\mathrm{THz}$ pulses in a molecular medium having resonant transitions in $\mathrm{THz}$ range. The pulses interact with the molecules coherently and do not overlap in the medium. The amplitude of the grating can be controlled either by tuning the pump amplitude or by an additional pulse. The grating modulation depth is proportional to the square of the $\mathrm{THz}$ field amplitude but is also influenced by the coherence times in the medium.

In the case of a multi-level system, using the first order of the perturbation theory in the weak-field approximation, we studied the grating dynamics created by two, three and four subcycle pulses. The possibility of a grating creation and their ultrafast control (erasing and spatial frequency multiplication) in multi-level system excited by subcycle pulses has been shown. A possible scheme of a setup for a grating formation and their control using four counter-propagating pulses on vibrations transitions in a $\mathrm{H}_{2} \mathrm{O}$ molecule without overlap in the medium was considered.

Our analysis revealed significant advantages of a grating creation in the $\mathrm{THz}$ range with respect to the optical range considered earlier. Because of high dipole moments in the $\mathrm{THz}$ range, one needs significantly smaller field amplitudes than in the optical range, of the order of $\mathrm{kV} / \mathrm{cm}$. This opens a new opportunity for an ultrafast $\mathrm{THz}$ spectroscopy with subcycle $\mathrm{THz}$ pulses to determine the resonance frequencies and the corresponding dephasing $T_{2}$ and relaxation $T_{1}$ times via diffraction of a weak probe pulse on the induced gratings ${ }^{12-14}$. The investigated phenomenon can be also used for creation of ultrafast beam deflectors and switches on molecular systems in the $\mathrm{THz}$ range.

As we have seen, somewhat counter-intuitively, the $\mathrm{HO}$ model in the perturbative regime provides lower excitation probabilities of the higher states than, for instance, the atomic potential $1 / r$, and thus the quality of the grating in the molecular case is better than in the case of an atom. This was supported also by non-perturbative simulations with the Bloch equations.

Numerical simulations performed for the parameters of $\mathrm{H}_{2} \mathrm{O}$ molecules and analytical results revealed that in the low-field case gratings have a harmonic shape. But in the strong-field case, when the perturbation approach is not valid anymore, the grating shape differs dramatically from the harmonic one. It has complex peak structure, since the level populations in the medium undergo multiple Rabi floppings over the pulse duration.

The obtained results also indicate a possibility of effective grating creation by quasi-unipolar subcycle $\mathrm{THz}$ pulses with respect to bipolar few-cycle ones. This efficient grating creation occurs in spite of the broad spectrum of the subcycle pulses covering many resonances at once, and non-resonant interaction with the molecule transitions. This result opens novel opportunities in ultrafast spectroscopy in the $\mathrm{THz}$ range with unipolar half-cycle pulses.

Received: 11 May 2020; Accepted: 4 January 2021

Published online: 21 January 2021 


\section{References}

1. Roskos, H. G., Thomson, M. D., Kress, M. \& Loeffler, T. Broadband THz emission from gas plasmas induced by femtosecond optical pulses: From fundamentals to applications. Laser Photon. Rev. 1, 349 (2007).

2. Reiman, K. Table-top sources of ultrashort THz pulses. Rep. Progr. Phys. 70, 1597 (2007).

3. Lepeshov, S., Gorodetsky, A., Krasnok, A., Rafailov, E. \& Belov, P. Enhancement of terahertz photoconductive antenna operation by optical nanoantennas. Laser Phot. Rev. 11(1), 1600199 (2016).

4. Fülöp, J. A., Tzortzakis, S. \& Kampfrath, T. Laser-driven strong-field terahertz sources. Adv. Opt. Mater. 8(3), 1900681 (2020).

5. Ferguson, B. \& Zhang, X. C. Materials for terahertz science and technology. Nat. Mater. 1(1), 26-33 (2002).

6. Jepsen, P. U., Cooke, D. G. \& Koch, M. Terahertz spectroscopy and imaging-Modern techniques and applications. Laser Photon. Rev. 5(1), 124-166 (2011).

7. Parrott, E. P. \& Zeitler, J. A. Terahertz time-domain and low-frequency Raman spectroscopy of organic materials. Appl. Spec. 69(1), $1-25(2015)$.

8. Zeitler, J. A. et al. Terahertz pulsed spectroscopy and imaging in the pharmaceutical setting-a review. J. Pharm. Pharmacol. 59, 209 (2007).

9. Williams, J. C. \& Twieg, R. J. Broadband terahertz characterization of the refractive index and absorption of some important polymeric and organic electro-optic materials. J. Appl. Phys. 109, 043505 (2011).

10. Murakami, H., Toyota, Y., Nishi, T. \& Nashima, S. Terahertz absorption spectroscopy of protein-containing reverse micellar solution. Chem. Phys. Lett. 519, 105 (2012).

11. Allen, L. \& Eberly, J. H. Optical Resonance and Two-level Atoms (Wiley, New York, 1975).

12. Abella, I. D., Kurnit, N. A. \& Hartmann, S. R. Photon echoes. Phys. Rev. 141, 391 (1966).

13. Shtyrkov, E. I., Lobkov, V. S. \& Yarmukhametov, N. G. Grating induces in ruby by interference of atomic states. JETP Lett. 27, 648 (1978).

14. Shtyrkov, E. I. Optical echo holography. Opt. Spectr. 114(1), 96 (2013).

15. Arkhipov, R. M. et al. Ultrafast creation and control of population density gratings via ultraslow polarization waves. Opt. Lett. 41, 4983 (2016).

16. Arkhipov, R. M. et al. Population density gratings induced by few-cycle optical pulses in a resonant medium. Sci. Rep. 7, 12467 (2017).

17. Arkhipov, R. M., Arkhipov, M. V., Pakhomov, A. V., Babushkin, I. \& Rosanov, N. N. Light-induced spatial gratings created by unipolar attosecond pulses coherently interacting with a resonant medium. Laser Phys. Lett. 14, 095402 (2017).

18. Arkhipov, R. M., Pakhomov, A. V., Arkhipov, M. V., Babushkin, I. \& Rosanov, N. N. Population difference gratings induced in a resonant medium by a pair of short terahertz nonoverlapping pulses. Opt. Spectrosc. 125(4), 586 (2018).

19. Eichler, H. J., Günter, P. \& Pohl, D. W. Laser-Induced Dynamic Gratings (Springer, Berlin, 1981).

20. Dolgaleva, K., Materikina, D. V., Boyd, R. W. \& Kozlov, S. A. Prediction of an extremely large nonlinear refractive index for crystals at terahertz frequencies. Phys. Rev. A 92, 023809 (2015).

21. Tcypkin, A. N. et al. High Kerr nonlinearity of water in THz spectral range. Opt. Express 27, 10419 (2019).

22. Kozlov, V. V., Rosanov, N. N., De Angelis, C. \& Wabnitz, S. Generation of unipolar pulses from nonunipolar optical pulses in a nonlinear medium. Phys. Rev. A 84, 023818 (2011).

23. Hassan, M. T. et al. Optical attosecond pulses and tracking the nonlinear response of bound electrons. Nature 530, 66 (2016).

24. Pakhomov, A. V. et al. All-optical control of unipolar pulse generation in a resonant medium with nonlinear field coupling. Phys. Rev. A 95, 013804 (2017).

25. Arkhipov, M. V. et al. Generation of unipolar half-cycle pulses via unusual reflection of a single-cycle pulse from an optically thin metallic or dielectric layer. Opt. Lett. 42, 2189 (2017).

26. Efimenko, E. S., Sychugin, S. A., Tsarev, M. V. \& Bakunov, M. I. Quasistatic precursors of ultrashort laser pulses in electro-optic crystals. Phys. Rev. A 98, 013842 (2018).

27. Tsarev, M. V. \& Bakunov, M. I. Tilted-pulse-front excitation of strong quasistatic precursors. Opt. Express 27, 5154 (2019).

28. Pakhomov, A. V. et al. Unusual terahertz waveforms from a resonant medium controlled by diffractive optical elements. Sc. Rep. 9, 7444 (2019).

29. Wu, H.-C. \& Meyer-ter Vehn, J. Giant half-cycle attosecond pulses. Nat. Photon. 6, 304 (2012).

30. Xu, J. et al. Terawatt-scale optical half-cycle attosecond pulses. Sci. Rep. 8, 2669 (2018).

31. Arkhipov, R. M., Arkhipov, M. V. \& Rosanov, N. N. Unipolar light: Existence, generation, propagation, and impact on microobjects. Quant. Electron. 50(9), 801 (2020).

32. Jones, R. R., You, D. \& Bucksbaum, P. H. Ionization of Rydberg atoms by subpicosecond half-cycle electromagnetic pulses. Phys. Rev. Lett. 70(9), 1236 (1993).

33. Wesdorp, C., Robicheaux, F. \& Noordam, L. D. Displacing rydberg electrons: The mono-cycle nature of half-cycle pulses. Phys. Rev. Lett. 87(8), 083001 (2001).

34. Wetzels, A. et al. Rydberg state ionization by half-cycle-pulse excitation: Strong kicks create slow electrons. Phys. Rev. Lett. 89(27), 273003 (2002).

35. Pan, Y., Zhao, S.-F. \& Zhou, X.-X. Generation of isolated sub-40-as pulses from gas-phase CO molecules using an intense few-cycle chirped laser and a unipolar pulse. Phys. Rev. A 87, 035805 (2013).

36. Chai, X. et al. Subcycle terahertz nonlinear optics. Phys. Rev. Lett. 121, 143901 (2018).

37. Arkhipov, R. M. et al. Unipolar subcycle pulse-driven nonresonant excitation of quantum systems. Opt. Lett. 44, 1202 (2019).

38. Arkhipov, R. M. et al. Selective ultrafast control of multi-level quantum systems by subcycle and unipolar pulses. Opt. Express 28(11), 17020-17034 (2020).

39. Rosanov, N. N. \& Vysotina, N. V. Direct acceleration of a charge in vacuum by linearly polarized radiation pulses. J. Exp. Theor. Phys. 130, 52-55 (2020).

40. Arkhipov, R. M., Arkhipov, M. V. \& Rosanov, N. N. On the possibility of holographic recording in the absence of coherence between a reference beam and a beam scattered by an object. JETP Lett. 111(9), 484-488 (2020).

41. Arkhipov, R. M., Arkhipov, M. V., Pakhomov, A. V. \& Rosanov, N. N. Population gratings produced in a quantum system by a pair of sub-cycle pulses. Quant. Electron. 49(10), 958 (2019).

42. Landau, L. D. \& Lifshitz, E. M. Quantum Mech. (Pergamon, Oxford, 1974).

43. Van Exter, M., Fattinger, C. \& Grischkowsky, D. Terahertz time-domain spectroscopy of water vapor. Opt. Lett. 14(20), 1128-1130 (1989).

44. Heyden, M. et al. Dissecting the THz spectrum of liquid water from first principles via correlations in time and space. Proc. Natl. Acad. Sci. 107(27), 12068-12073 (2010).

45. Lee, Y.S. Terahertz Spectroscopy of Atoms and Molecules, In: Principles of Terahertz Science and Technology. Springer, Boston, MA (2009).

46. Zhang, H. et al. Autler-Townes splitting of a cascade system in ultracold cesium Rydberg atoms. Phys. Rev. A 87(3), 033835 (2013).

47. Bednarek, S., Szafran, B. \& Adamowski, J. Theoretical description of electronic properties of vertical gated quantum dots. Phys. Rev. B 64, 195303 (2001).

48. Borri, P. et al. Ultralong dephasing time in InGaAs quantum dots. Phys. Rev. Lett. 87(15), 157401 (2001). 
49. Bayer, M. \& Forchel, A. Temperature dependence of the exciton homogeneous linewidth in In060 Ga 040 As/GaAs self-assembled quantum dots. Phys. Rev. B 65(4), 041308 (2002).

50. Leyman, R. R. et al. Quantum dot materials for terahertz generation applications. Laser Photon. Rev. 10(5), 772-779 (2016).

51. Scalari, G. et al. THz and sub-THz quantum cascade lasers. Laser Photon. Rev. 3, 45-66 (2009).

52. Choi, H. et al. Time-resolved investigations of electronic transport dynamics in quantum cascade lasers based on diagonal lasing transition. IEEE J. Quantum Electron. 45, 307-321 (2009).

53. Choi, H. et al. Ultrafast Rabi flopping and coherent pulse propagation in a quantum cascade laser. Nat. Photon. 4(10), 706-710 (2010).

54. Babbit, W. R. \& Mossberg, T. Time-domain frequency-selective optical data storage in a solid-state material. Opt. Commun. 65, 185 (1988).

55. Akhmanov, S. A. \& Nikitin, S. Y. Physical Optics (Clarendon Press, Oxford, 1997).

56. Rosanov, N. N., Arkhipov, R. M. \& Arkhipov, M. V. On laws of conservation in the electrodynamics of continuous media (on the occasion of the 100th anniversary of the SI Vavilov State Optical Institute). Physics-Uspekhi 61(12), 1227 (2018).

57. Maier, M. Applications of stimulated Raman scattering. Appl. Phys. 11(3), 209-231 (1976).

58. Akhmanov, S. A. \& Koroteev, N. I. Methods of Nonlinear Optics in Light Scattering Spectroscopy (Nauka, Moscow, 1981).

59. Born, M. \& Wolf, E. Principles of Optics: Electromagnetic Theory of Propagation, Interference and Diffraction of Light (Elsevier, Amsterdam, 2013).

\section{Author contributions}

R. A. and A. P. provided analytical theory, R. A., A. P., I. B. and M. A. performed numerical simulations, I. B., A. D., U. M. and N. R. analysed the results and participated in the discussions, article formulation and writing. All authors reviewed the manuscript.

\section{Funding}

Open Access funding enabled and organized by Projekt DEAL. R.A. thanks Russian Science Foundation, project 19-72-00012 for the financial support (analytical and numerical study of gratings formation). I.B., U.M. and A.D. are thankful to the Deutsche Forscunggemeinschaft (DFG) projects BA 4156/4-2, MO 850-19/2 and MO 850-23/1, as well as to Germany's Excellence Strategy within the Cluster of Excellence PhoenixD (EXC 2122, Project ID 390833453).

\section{Competing interests}

The authors declare no competing interests.

\section{Additional information}

Correspondence and requests for materials should be addressed to I.B.

Reprints and permissions information is available at www.nature.com/reprints.

Publisher's note Springer Nature remains neutral with regard to jurisdictional claims in published maps and institutional affiliations.

(1) Open Access This article is licensed under a Creative Commons Attribution 4.0 International License, which permits use, sharing, adaptation, distribution and reproduction in any medium or format, as long as you give appropriate credit to the original author(s) and the source, provide a link to the Creative Commons licence, and indicate if changes were made. The images or other third party material in this article are included in the article's Creative Commons licence, unless indicated otherwise in a credit line to the material. If material is not included in the article's Creative Commons licence and your intended use is not permitted by statutory regulation or exceeds the permitted use, you will need to obtain permission directly from the copyright holder. To view a copy of this licence, visit http://creativecommons.org/licenses/by/4.0/.

(c) The Author(s) 2021 